\title{
GEOGRAFÍA DE LA INFANCIA: ESPACIOS DE JUEGO EN CIUDADES MEDIAS DE CATALUÑA*
}

\author{
Mireia Baylina Ferré, Anna Ortiz Guitart y María Prats Ferret \\ Dpto. de Geografía, Universidad Autónoma de Barcelona, 08193 Bellaterra \\ Mireia.Baylina@uab.es; Anna.Ortiz@uab.es; Maria.Prats@uab.es
}

\begin{abstract}
Resumen: Este artículo tiene como objetivo reflexionar sobre el papel de los espacios de juego en la vida cotidiana de los niños y niñas en entornos urbanos, conocer el tipo de utilización y apropiación que hacen de estos espacios y ver hasta qué punto se puede considerar el género como uno de los elementos organizadores de los espacios de juegos. El trabajo se contextualiza en dos municipios medios catalanes, Manresa y Sant Feliu de Llobregat, capitales comarcales del Bages y el Baix Llobregat respectivamente, ambas localizadas en la provincia de Barcelona.
\end{abstract}

Palabras clave: infancia, espacios de juego, género, Catalunya.

\begin{abstract}
This article aims to explore the role of playgrounds in children's daily life in urban environements, to know which is the use of these spaces and to see up to which point gender may be considered the organizing element in these areas. The research context is two medium-size catalan towns, Manresa and Sant Feliu de Llobregat, county capitals of Bages and Baix Llobregat respectively, both located in Barcelona's province.
\end{abstract}

Key words: childhood, playgrounds, gender, Catalunya.

"Los niños/as deberían tener el mismo espacio para jugar que el que tienen los adultos/as para estacionar" (Tonucci, 2004).

Este artículo pretende reflexionar sobre el papel de los espacios de juego en la vida cotidiana de los niños y niñas de dos ciudades medias de Catalunya, Manresa y Sant Feliu de Llobregat. Concretamente, se desea conocer qué tipo de utilización y apropiación hacen de estos espacios y ver hasta qué punto se puede considerar el

* Recibido: 4-10-2005. Aceptado: 8-1-2007. 
género como uno de los elementos organizadores de los espacios de juegos. Es importante tener en cuenta la contextualización del trabajo en estas ciudades, ya que la experiencia de la infancia no es universal sino una construcción social. Así, los resultados de esta investigación deben entenderse dentro del contexto de las sociedades occidentales, donde la infancia es vista como un tiempo de inocencia, vulnerabilidad y dependencia, muy distinta al tipo de infancia experimentada en otras sociedades donde las experiencias de pobreza, indigencia o enfermedad de sus padres obligan a los niños y niñas a entrar, sin contemplaciones, al mundo de los adultos.

\section{Las geografías personales de la infancia}

En los últimos años han aumentado el número de publicaciones sobre la llamada geografía de la infancia interesada en el análisis de la vida cotidiana de los niños y niñas ${ }^{1} \mathrm{y}$, muy especialmente, en su comportamiento y presencia en los espacios públicos urbanos. Desde la geografía social (Tandy, 1999), cultural (Matthews, 1995) y del género (Karsten, 2003; Valentine, 1996), se han ido produciendo numerosas investigaciones preocupadas por visibilizar la diversidad de experiencias y necesidades de los colectivos de personas que, por cuestiones de género, edad, sexualidad y condición social, cultural y étnica han quedado tradicionalmente excluidos de los estudios urbanos.

En relación con el estudio del uso y la apropiación de los espacios públicos por parte de niños y niñas se ha observado como su presencia es cada vez menor debido a una serie de razones: en primer lugar, los niños dedican más tiempo a mirar la televisión o a jugar con el ordenador de su casa (Karsten, 1998); en segundo lugar, la disminución del número de hijos provoca que haya más posibilidades económicas para ofrecerles una educación más "correcta", materializada a través de las actividades extraescolares en espacios semipúblicos (Droogleever; Karsten, 1999); y, en tercer lugar, el espacio público se convierte, cada vez más, "naturalmente" y "normalmente", en un espacio de adultos, de forma que los padres y particularmente las madres son conscientes de los riesgos y posibles peligros de los espacios públicos, por lo cual restringen el uso a sus hijos (Valentine, 1997). Respecto a este último punto cabe señalar que mientras la identidad de los niños se construye sobre el imaginario de su "vulnerabilidad" en los espacios públicos, estadísticamente se demues-

\footnotetext{
${ }^{1}$ A pesar de nuestra preocupación por el uso de un lenguaje inclusivo y no sexista y a petición de los editores de la revista, en adelante utilizaremos preferentemente el genérico "niños" para referirnos al conjunto de niños y niñas a fin de hacer más ligera la lectura del texto. Procuraremos, sin embargo, mantener expresiones más inclusivas como por ejemplo "infancia" cuando el contexto de la frase lo permita.
} 
tra como éstos (del mismo modo que los adultos) sufren más agresiones en el espacio privado y que éstas son a la vez, y paradójicamente, provocadas por personas de su mismo ámbito familiar. Según Valentine (2004), los miedos que los padres sienten por la seguridad de sus hijos en el espacio público son construidos y mediatizados, en cierta medida, por los medios de comunicación, las campañas educativas y la comunidad donde viven. Los mensajes que estos sectores generan contribuyen a presuponer que el espacio público sea "naturalmente" un espacio para adultos y, consecuentemente, un espacio donde los niños viven permanentemente bajo el riesgo de caer en manos de personas extrañas. Estos "extraños" son demonizados a través del cuerpo masculino que pasa a ser concebido como un cuerpo potencialmente peligroso y desafiante, mientras que el cuerpo femenino continua siendo concebido como un cuerpo transmisor de seguridad y confianza.

Numerosas geógrafas han reflexionado sobre las consecuencias que tienen las actitudes extremadamente protectoras de los padres en relación al uso que niños y niñas hacen de los espacios públicos. Se observa como cada vez más el tiempo libre de los niños transcurre en espacios privados y semipúblicos (en la propia casa, en centros deportivos, en ludotecas, etc.) y como, consecuentemente, las actividades que desarrollan éstos están cada vez más privatizadas, institucionalizadas y organizadas por los adultos (McKendrick et al., 2000; Gagen, 2000; Valentine, 2004). Algunos estudios muestran como el hecho de que los niños no puedan acceder al espacio urbano que los rodea solos o jugar en los espacios públicos abiertos sin la mirada atenta de un adulto tiene repercusiones en el desarrollo de la autoestima, la responsabilidad y la independencia de los niños (Román, 1995, 2000). Se observa también como esta privatización del tiempo libre comporta a la vez una mayor segregación social en el tiempo libre de los niños. En relación a este hecho, Karsten (2002) muestra como el tiempo libre de los hijos de familias de ingresos medios-altos con transporte privado se desarrolla fuera de los espacios públicos, mientras que el tiempo libre de los hijos de familias de ingresos bajos transcurre al aire libre, jugando en los espacios públicos abiertos del entorno cotidiano.

Las diferencias de género son visibles cuando se observa como los niños y las niñas usan el área de juegos de los espacios públicos. Según los resultados empíricos de Karsten (1998; 2003), en barrios multiculturales de Ámsterdam, la presencia de niñas en el área de juegos es menor que la de los niños, así como el tiempo que pasan en él y el espacio que utilizan para jugar. Diversos autores señalan el importante papel que tienen las áreas de juegos infantiles en la planificación urbanística y el diseño de los espacios públicos para construir ciudades que tengan más en cuenta las necesidades específicas de los niños (Borja; Castells, 1997); también señalan la necesidad de favorecer la participación de los niños y los jóvenes en el diseño de los espacios públicos (Simpson, 1997) y animar la presencia de las niñas en las áreas de juegos infantiles (Paravicini, 2000). Respecto a este último punto, Karsten (2003) apunta algunas recomendaciones para el diseño de estas áreas: deben ser suficiente- 
mente grandes para facilitar los juegos de ambos sexos; el espacio dedicado al equipamiento (columpios, toboganes, etc.) tiene que ser equivalente al dedicado al área concebida para los juegos de pelota y, finalmente, tiene que haber un buen mantenimiento del área de juegos y una adecuada renovación de los equipamientos.

\section{Metodología y caracterización de las áreas de estudio}

La realización de esta investigación se fundamenta en la metodología cualitativa a partir de observaciones directas, entrevistas informativas a expertos y breves entrevistas a usuarios. Esta metodología ya ha sido experimentada con anterioridad por las autoras en estudios sobre espacios urbanos en la ciudad de Barcelona (Ortiz, 2004). Durante los meses de junio y julio de 2004 se llevaron a cabo varias observaciones (dos en días laborables y dos en días festivos y a diferentes horas del día) que permitieron cartografiar, según las variables de sexo y grupos de edad, el uso de dos espacios públicos en cada una de las ciudades escogidas para el análisis. Los mapas de uso y de apropiación (ver Mapa 1) tienen como objetivo ilustrar el número de personas que usan el espacio público, su localización dentro del espacio, así como las interrelaciones entre las personas y las actividades desarrolladas en dicho espacio.

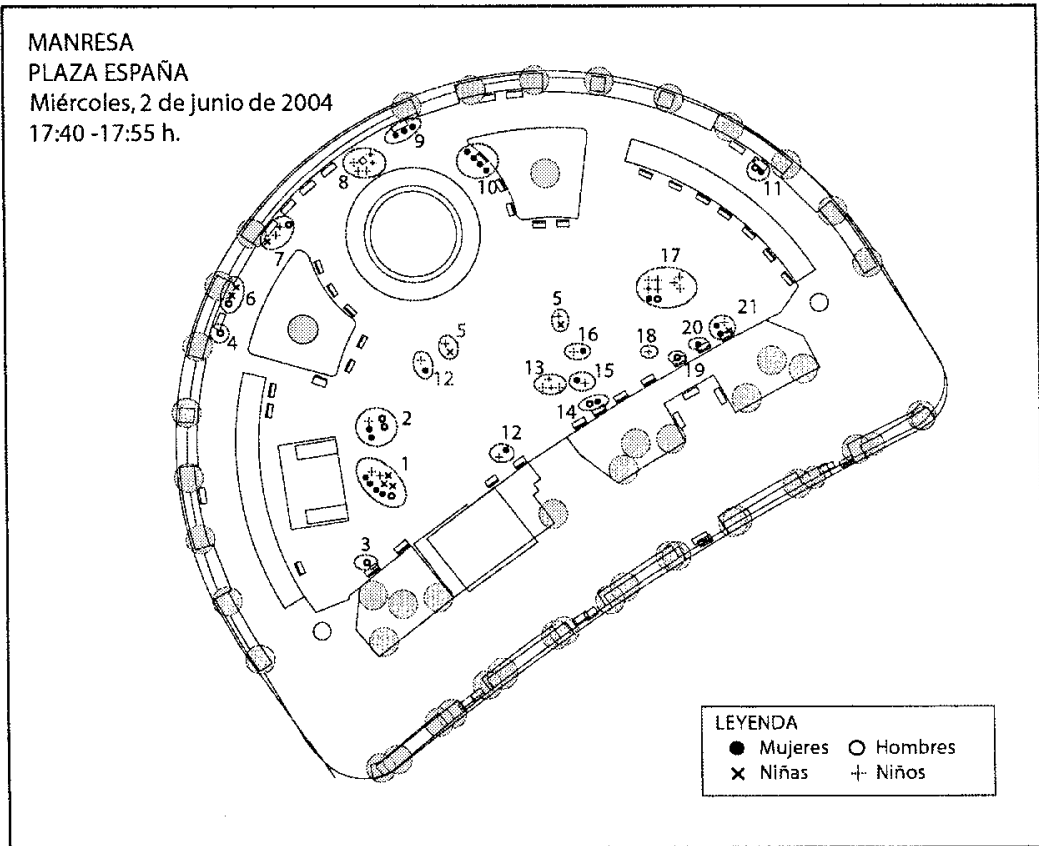


Estas observaciones pretendian captar detalladamente la vida en el espacio público poniendo especial énfasis en la descripción del uso (número y perfil de las personas) y de la apropiación (conjunto de interrelaciones entre personas y actividades, individuales o colectivas, desarrolladas) de los usuarios. Paralelamente se realizaron sobre el terreno breves entrevistas a niños y a personas adultas (madres, padres, abuelas, cuidadoras, etc.) que les acompañaban en su tiempo libre.

La información se ha complementado con entrevistas informativas a personas expertas (concejales de urbanismo, educación e infancia; así como, arquitectos y planificadores urbanos), para obtener opiniones cualificadas sobre las transformaciones físicas y sociales de las ciudades y los espacios públicos estudiados durante los últimos años.

El análisis se ha llevado a cabo en Manresa y Sant Feliu de Llobregat; ambas ciudades son cabeceras de comarca relativamente significativas en el contexto demográfico y económico catalán. Se han considerado cuatro espacios de juego, dos en cada ciudad. Uno de ellos ubicado en el centro histórico (Plaza España en Manresa y Plaza Lluís Companys en Sant Feliu de Llobregat) y otro en la periferia, en un barrio de reciente creación (Plaza Bases y Parque Europa respectivamente). Hemos hecho esta distinción entre dos tipos de barrio porque consideramos que las dinámicas sociales y de uso, así como las características del diseño de los espacios y su ubicación y contexto urbano difieren suficientemente como para permitir la comparación y ver como incluso dentro de una misma ciudad conviven varios modelos de espacios de juego.

\subsection{Los espacios del centro de la ciudad: Plaza España y Plaza Lluís Companys}

\subsubsection{Plaza España: concurrencia y diversidad social y étnica}

La Plaza España (Foto 1) se encuentra en el centro de la ciudad de Manresa, concretamente en la zona de ensanche construida entre la segunda mitad del siglo XIX y los inicios del siglo XX. Esta plaza otorgó una nueva centralidad a la ciudad y incorporó una zona de juegos infantiles, de recreo para los estudiantes y de descanso para los paseantes.

La plaza, de unos $5.000 \mathrm{~m}^{2}$, es semicircular. En su lado lineal limita con la avenida y con un bar-terraza, y en el circular con la calzada, una zona de tráfico intenso y rodeado de servicios públicos como correos, un centro de asistencia primaria, un instituto de enseñanza secundaria, la comisaría de policía y dos paradas de autobuses.

Se trata de una plaza de sablón salpicada por elementos vegetales, una fuente, bancos, farolas, papeleras y juegos infantiles (torres, toboganes, casitas, muelles...). 


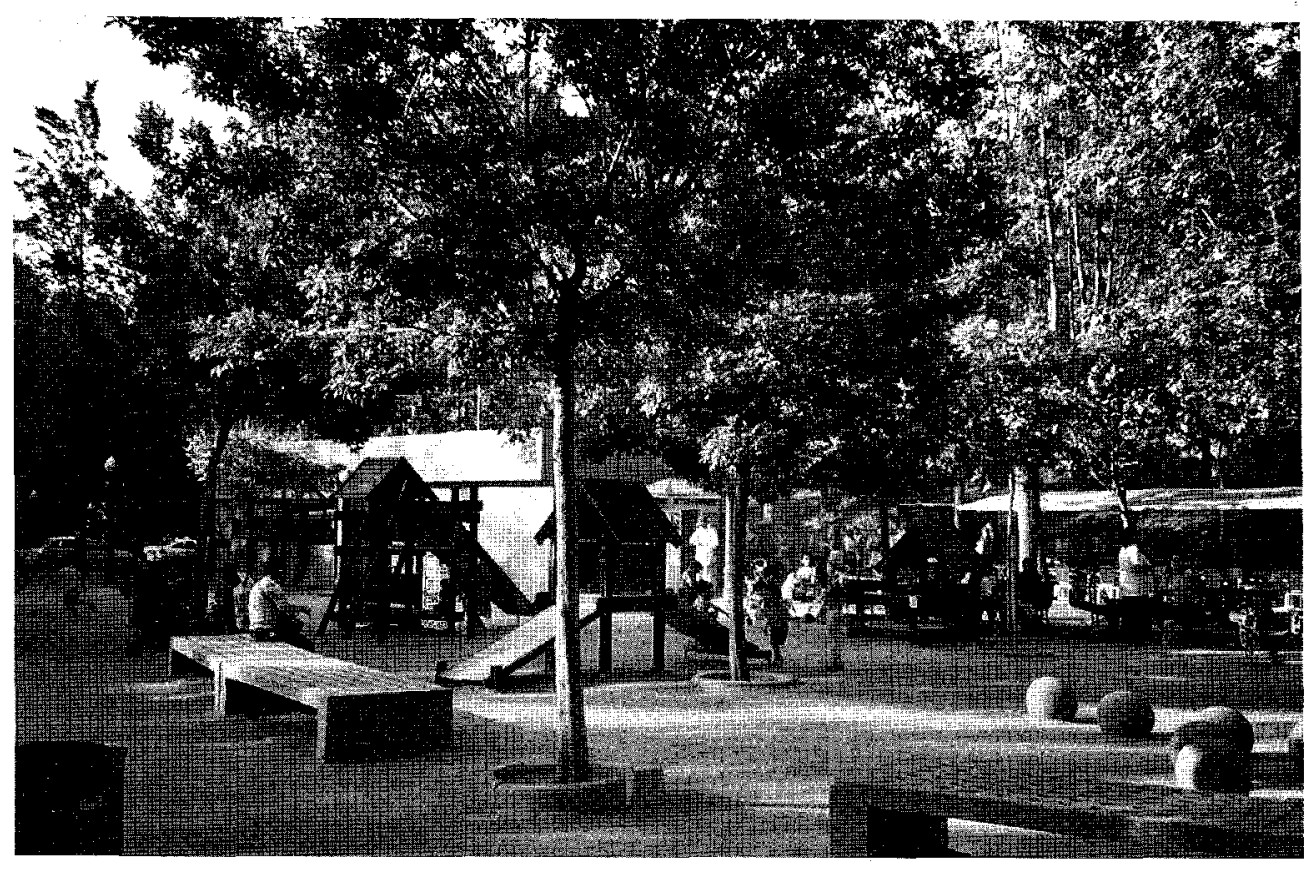

Foto 1. Plaza España (junio, 2004).

La disposición de los elementos muestra tres subespacios: uno de juegos infantiles; otro alrededor de la fuente, libre de juegos y con bancos alrededor; y un tercero que forma un arco con una hilera de bancos que miran al interior. Hay cinco accesos a la plaza, todos practicables para todas las personas; tres de principales por la parte que da a la avenida, abiertos y llanos y otras dos vías de entrada en el lado circular.

Las características más evidentes del uso de este espacio son la elevada concurrencia, tanto en días laborales como festivos, y la diversidad étnica y social en la población usuaria. Ello se explica por su centralidad, su fácil acceso desde la avenida y por la relativa escasez de espacios similares alrededor. La plaza ofrece una sensación de movimiento y de gran variedad de personas en relación al género, edad, origen cultural y posición social.

\subsubsection{Plaza Lluís Companys: un espacio vital de itinerario cotidiano}

El rasgo más característico de este espacio (Foto 2) es su localización en pleno centro histórico de la ciudad de Sant Feliu de Llobregat. A pesar de ello es un espa- 


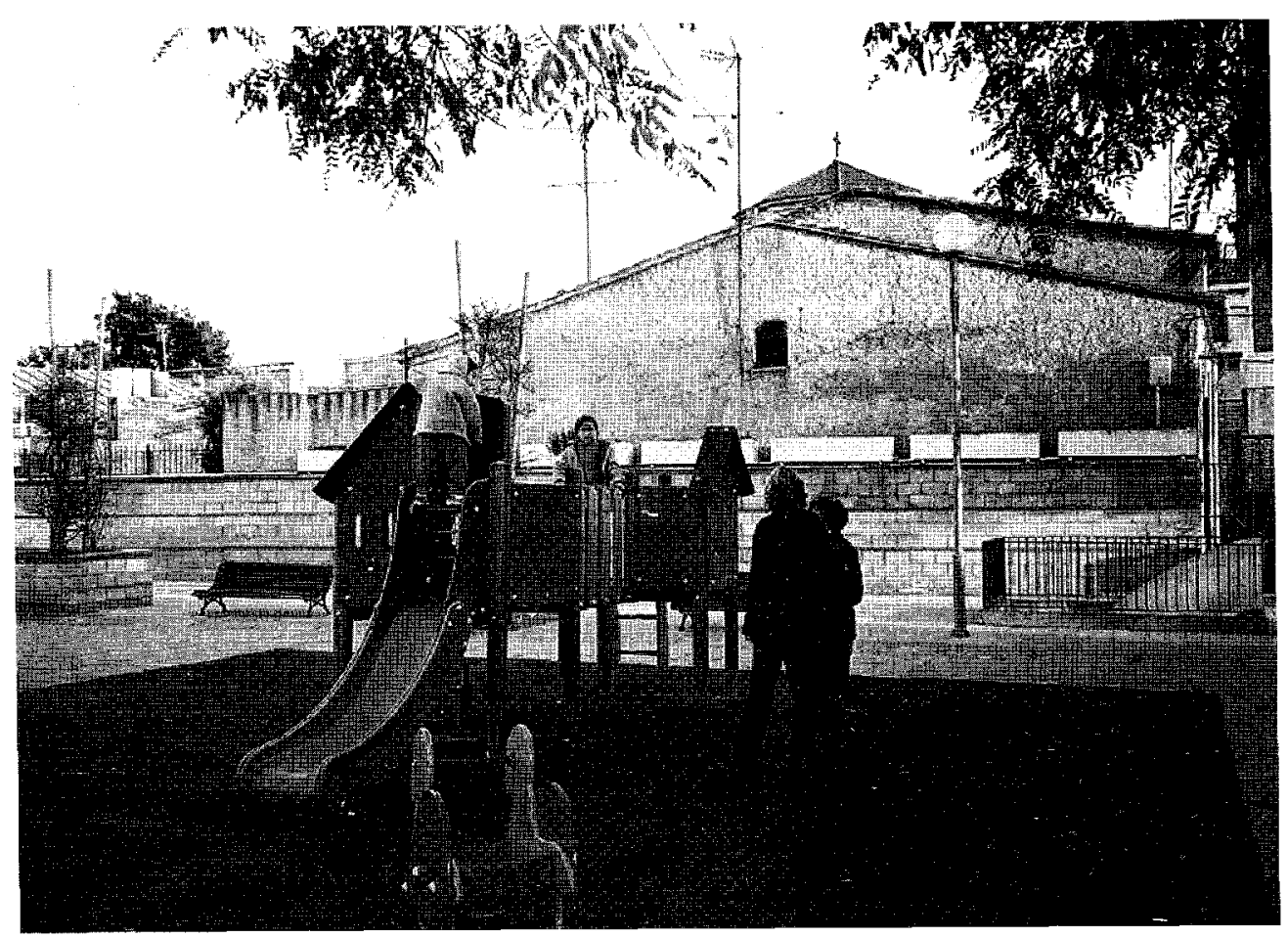

Foto 2. Plaza Lluís Companys (marzo, 2005).

cio reciente; se crea a inicios de los años 90, a raíz de la construcción de un aparcamiento subterráneo que implicó la cesión de la superficie para uso público. Este particular origen del espacio, urbanizado inicialmente por el promotor del aparcamiento, es responsable de algunas de las limitaciones que presenta. Poco después los Servicios Técnicos Municipales realizaron un proyecto de mejoras de la Plaza que la dotó de algunos de los escasos elementos que hoy la configuran.

El aspecto que presenta actualmente nos permite definirla como una plaza dura, es decir pavimentada y con escasas posibilidades de convertirla en un espacio con vegetación al encontrarse sobre la cubierta impermeable del aparcamiento. En una superficie de aproximadamente $3.000 \mathrm{~m} 2$ se dispone de dos grupos de bancos orientados hacia el centro de la Plaza. Encontramos también una pérgola metálica con vegetación, bajo la cual se localiza un banco corrido de piedra. Junto a los demás bancos encontramos maceteros alli donde no es posible plantar en el suelo.

El espacio central se subdivide en dos, situándose una fuente entre ambos: un pequeño espacio con pavimento blando, con juegos dirigidos a los niños más peque- 
ños y una pista consistente en un gran espacio liso, para los chicos más mayores, que suele destinarse a los juegos de pelota, a patinar o a ir en bicicleta.

El acceso a esta plaza se realiza desde dos calles por medio de rampa y/o escalera. Una característica resaltable es que se trata de una Plaza con verjas metálicas que se cierran por la noche, preservándola de otros usos. Una antigua masía, actualmente de propiedad municipal y reconvertida para usos culturales rodea la Plaza por uno de los lados, el resto de edificios alrededor son viviendas. Finalmente cabe señalar que este es un espacio de gran significación ciudadana como espacio festivo, ya que durante las Fiestas patronales acoge numerosos actos y espectáculos.

La Plaza Lluís Companys es muy valorada por su centralidad y por formar parte de los itinerarios cotidianos de muchos habitantes, principalmente en el marco de los itinerarios escolares. La centralidad de esta Plaza implica que se encuentra en la zona de edificación más antigua y más densa de la ciudad, donde los espacios abiertos y las zonas verdes escasean más que en los barrios de más reciente creación. La escasez de alternativas en esta parte de la ciudad también contribuye a una ocupación bastante alta para un espacio con estándares de calidad relativamente bajos.

\subsection{Las nuevas periferias: amplios espacios para una población joven}

\subsubsection{Plaza Bases}

La Plaza Bases (Foto 3) se encuentra un barrio creado a mediados del siglo XX para albergar a población recién llegada a Manresa. Sin embargo, la plaza y su entorno inmediato han permanecido sin urbanizar hasta el año 2001, en que un plan parcial de promoción privada permitió la construcción del espacio viario. La plaza Bases ocupa una posición central en la zona y sirve mayoritariamente para la población residente en el área, básicamente parejas jóvenes que buscan en esta zona una mejor calidad de vida.

La plaza se encuentra circundada por cuatro viales y se trata de un espacio abierto de unos $9.000 \mathrm{~m}^{2}$, de forma triangular y con un destacado desnivel de norte a sur. Un $60 \%$ del espacio está formado por césped y pinos y su superficie es ondulada. El lado oeste y sur, formando una franja alargada, está orientado al juego y forma tres subespacios: dos zonas de petanca con pavimento de sablón, una fuente y dos hileras de bancos; en el centro, un espacio vacío de elementos, excepto bancos; y al sur, una zona infantil, con arenal, estructuras de juegos (tren, casa, puente, columpios y muelles) y algunos bancos. La vegetación de la plaza está formada por césped y algunos árboles repartidos por todo el espacio. 


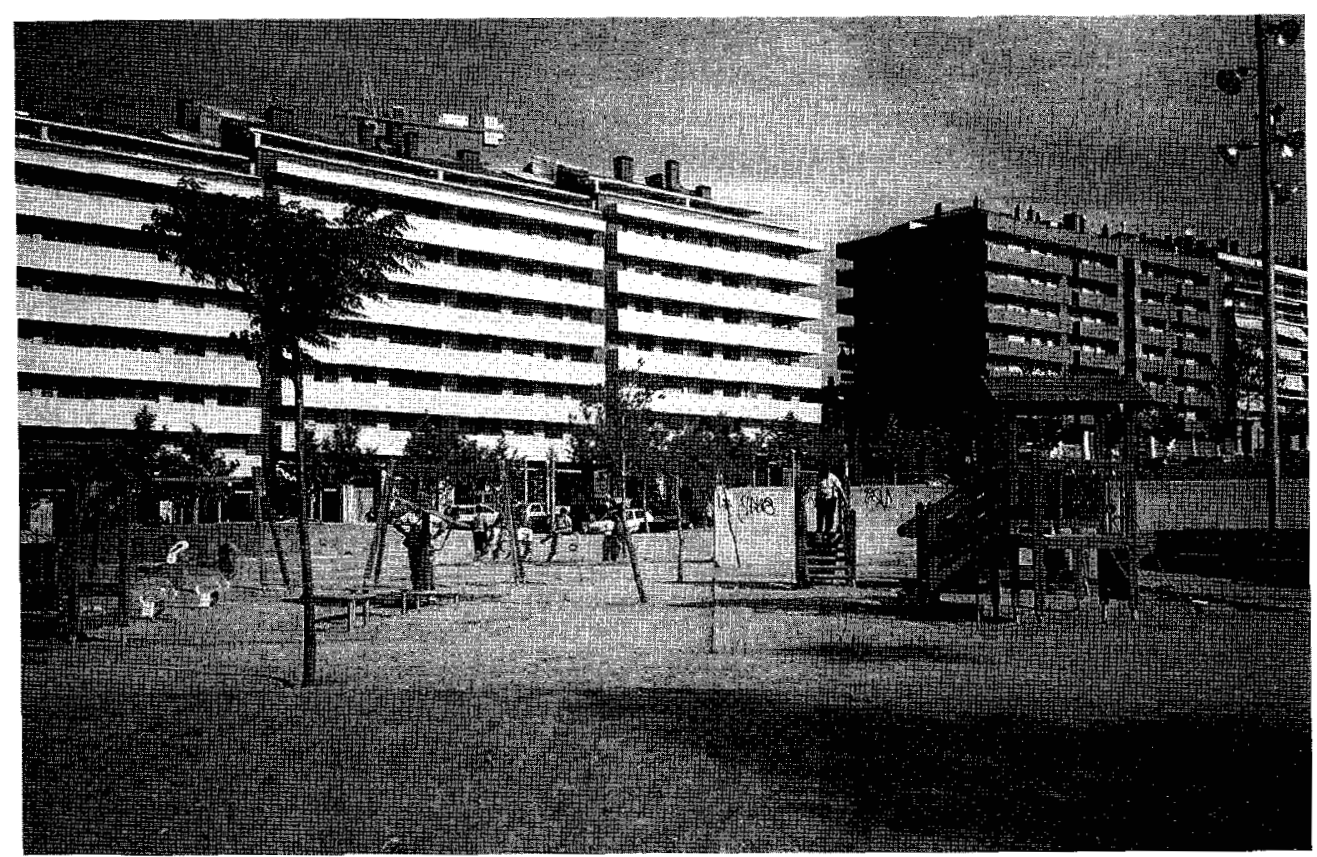

Foto 3. Plaza Bases (junio, 2004).

Hay cuatro accesos claramente definidos y tres de ellos son practicables. Dos se encuentran en el área de juegos infantiles; otro se encuentra en la zona de juego central; y el último se encuentra en la parte superior. La zona de juegos infantiles es quizás la de más difícil acceso, ya que tiene bastante pendiente y está muy cerca de la rotonda. En esta área, y a pesar de los dos accesos existentes, los usuarios se han apropiado del espacio improvisando una nueva vía para acceder al espacio cruzando directamente el parterre. La iluminación es abundante en toda la zona.

El grado de utilización de la plaza es moderado, algo que puede deberse a su posición periférica respecto al centro de la ciudad, a tratarse de un espacio relativamente nuevo y por tanto aún poco conocido, o a la competencia de otras áreas de juego cerca y a la existencia de jardines privados.

\subsubsection{Parque Europa}

El origen del Parque Europa (Fotografía 4) es también muy reciente, ya que fue inaugurado a finales de 1997. Está asociado al proyecto de urbanización del plan par- 


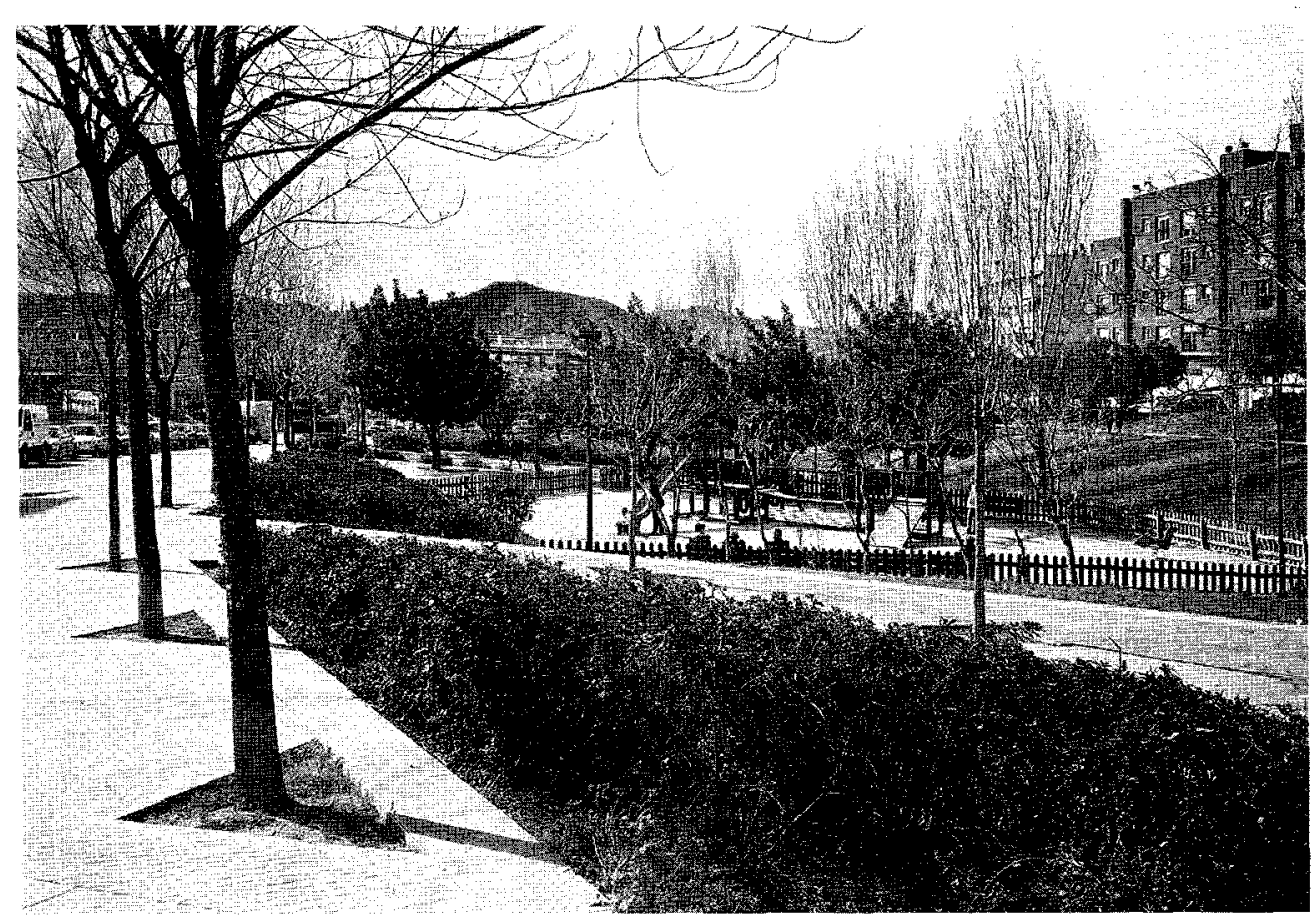

Foto 4. Parque Europa (marzo, 2005).

cial del sector de Mas Lluí, un barrio de nueva creación en la ciudad de Sant Feliu de Llobregat, que ha supuesto la edificación de 1800 viviendas de variada tipología. Este nuevo barrio ha sido edificado con criterios de calidad, intentando no densificar el territorio y creando zonas verdes comunitarias en el interior de las manzanas. Este factor es importante a la hora de analizar el grado de ocupación del Parque estudiado, ya que esta existencia de zonas verdes alternativas, es decir de una mayor oferta, redunda en una menor frecuentación relativa de los espacios verdes públicos disponibles.

El Parque Europa se asienta sobre el cauce de un antiguo torrente actualmente canalizado, y su trazado se adapta a las características del terreno. El diseño prevé diversos usos, para los cuales se han previsto varios espacios en una sucesión de plataformas llanas, acondicionadas con distintos elementos. Por ejemplo, encontramos plataformas con bancos para el reposo, la observación y la conversación, plataformas con juegos infantiles e incluso plataformas sin más elementos que la vegetación, pero que también son usadas por la población 
En la parte más inclinada del terreno encontramos un bosque claro plantado con especies locales, así como variados arbustos y matorrales. Recorriendo este espacio ajardinado se ha trazado un sendero que permite recorridos peatonales alternativos al viario. En el tramo situado más al norte el Parque se ensancha y termina en un estanque artificial donde se encuentran patos y ocas, un lugar muy apreciado por la población infantil, que acude a contemplar los animales. El acceso al Parque se puede realizar desde numerosos puntos, ya que no existe separación física ni vallado entre el espacio del Parque y las calles adyacentes.

\section{La vida cotidiana en los espacios de juego: valoración positiva y uso intenso}

En términos generales, se considera que la oferta de espacios de juegos es suficiente, aunque quizá no plenamente adecuada para todos los usuarios potenciales. Así por ejemplo, se menciona a menudo el hecho de que los espacios están pensados para un público muy limitado: la franja central de la edad infantil, entre 3 y 10 años aproximadamente, pero en cambio los espacios y juegos propuestos son poco adecuados para los bebés, así como para los niños mayores de 10 años y especialmente para los adolescentes. Esta franja de edad, por ejemplo, no encuentra en los espacios de juego la posibilidad de esconderse, practicar deportes o simplemente jugar a la pelota, sea porque el espacio es muy pequeño, porque esta práctica no es compatible con la presencia de niños pequeños o personas mayores o simplemente por el hecho de que se prohíbe explícitamente. La apértura de los patios de las escuelas para uso vecinal, fuera del horario escolar, es una de las vías apuntadas para dar respuesta a esta demanda insatisfecha (Tonucci, 2004).

En general las zonas más ocupadas en los espacios públicos estudiados son los espacios de juegos infantiles, las zonas dotadas de algún tipo de mobiliario urbano y muy particularmente de estructuras de juegos, por lo que la presencia de bebés y niños hasta los 10 años, así como de sus acompañantes es muy destacada. En cambio las áreas más vacías de elementos son también las más vacías de personas. Cuando existe un espacio que lo permite, por ejemplo una pista asfaltada, encontramos también niños más mayores que van en bicicleta, corren, juegan a pelota o juegan con el agua de las fuentes. Eventualmente las zonas contiguas o alrededor del espacio de juegos son ocupadas por personas adultas, ancianas (sobre todo hombres) y/o por jóvenes o adolescentes, que utilizan los bancos o el césped para descansar.

Las diferencias temporales más destacables en el uso de los espacios de juego se producen entre días laborables y festivos. Durante los días laborables este espacio es 
muy utilizado por los niños y por las personas que están a su cargo; es, por tanto, fuera del horario escolar cuando encontramos la máxima ocupación, especialmente por las tardes. En los días festivos la ocupación es más dispersa a lo largo del día, aunque también es ligeramente más numerosa por las tardes que por las mañanas.

La utilización de los espacios según género es bastante igualitaria, incluso en el área de juegos. La presencia de niños y niñas en el espacio es bastante equilibrada, especialmente entre el grupo de menor edad, donde no se observan diferencias ni en el número, ni en el tipo de actividad desarrollada.

Sin embargo con la edad también aumenta la segregación de niños y niñas según el tipo de juego. Se observa que la realización de determinadas actividades conduce a un sesgo de género bastante claro: por ejemplo cuando se juega a pelota o concretamente a fútbol, una actividad que consume y monopoliza una gran cantidad de espacio, la presencia masculina es abrumadoramente mayoritaria. En general se constata que los niños hacen un uso más extensivo del espacio que las niñas. La actividad de patinaje, en cambio, es más frecuentemente realizada por niñas, aunque también algunos niños participan. Uno de los espacios que destaca por su clara feminización es el columpio, donde encontramos una mayoría de niñas, especialmente a medida que crece la edad. El escondite o el juego alrededor del agua de la fuente interesa a todos/as: pistolas de agua, globos hinchados de agua... En la estructura de juegos, donde es posible encaramarse, hacer equilibrios y tirarse por un tobogán, la presencia de niños y niñas es bastante equilibrada.

En cuanto a las personas acompañantes se observa que suelen ser mujeres (abuelas, hermanas, canguros, etc.). En cualquier caso, siempre hay más madres que padres, especialmente los dias laborables. Los padres solos aparecen en mayor medida las mañanas de los fines de semana.

La edad de los usuarios se configura como un elemento central en la estructuración y la organización del espacio y se refleja en el uso y en las actividades desarrolladas, que suelen ser muy diferenciadas por grupos de edad. Los más pequeños juegan con el mobiliario infantil y alrededor de éste; entre este grupo se da poca variedad de actividades y escasa interacción, tanto por la edad, como también porque el mobiliario de juego es bastante limitado y repetitivo.

A medida que crece la edad de los niños y disminuye la oferta, encontramos una mayor diversificación de actividades, más actividad grupal y también mayor creatividad. Estos niños más mayores se alejan más de las zonas de material de juego y crean su propia forma de diversión, tienden más a inventar sus juegos. 


\section{La atención al entorno ambiental}

Uno de los temas que preocupan tanto a los usuarios como a los expertos es la poca atención al mantenimiento de los espacios de juego. En concreto hay quejas respecto al arreglo o reposición del mobiliario estropeado, conservación del suelo, suciedad provocada por los perros, pintadas en las paredes y la falta de conservación del ajardinamiento. Existe la idea de que "se crean los espacios y se dejan estar" y los mismos expertos lo reconocen. Sin embargo, también se expresa la falta de civismo de la población a la hora de ensuciar o pasear a los perros en lugares prohibidos. En cualquier caso, se pone en evidencia la poca cultura de mantenimiento que existe todavía en nuestras sociedades.

"Está un poco abandonado. En algunos columpios hay agujeros y huecos en el suelo. El mantenimiento es deficitario. No me gusta decirlo pero es asî." (responsable de Educación, mujer, 48 años).

"Se limpia poco; poco mantenimiento. Es muy caro. Los niños se quejan. Se quejan de cosas muy razonables como de la falta de luz, de agua y de las pintadas en las paredes." (técnica de Infancia, mujer, 63 años).

El ruido, los humos y la intensidad viaria, es un tema más destacado por los expertos que por los usuarios. Para éstos últimos, el tráfico es contemplado más como potencial peligro que como un riesgo ambiental, algo que no deja de sorprender cuando son los niños quienes están más expuestos a los polucionantes.

"Hay un nivel de contaminación acústica brutal; eso no tendría que darse en un espacio de juego para niños." (responsable local de Educación, mujer, 48 años).

Los lugares de sombra y de sol son básicos para los usuarios. En verano las sombras son imprescindibles y en invierno se busca el sol, y muchas de las observaciones que hacen son sobre si hay o no espacios sombreados o la correcta ubicación de los elementos que dan sombra. Alguna otra queja viene de la funcionalidad del diseño. Por ejemplo las fuentes, que pueden generar charcos de agua innecesariamente.

Por lo demás, los usuarios se consideran bastante satisfechos con estos espacios de juego. Sobre todo valoran la disponibilidad y la accesibilidad, y esto prima delante de algún otro descontento como una escasa vegetación, una plaza dura o un diseño estándar. En general, son espacios ambientalmente agradables, sobre todo los de reciente construcción ya que se ubican en entornos menos densos. La cuestión de la calidad ambiental es muy importante por diversas razones. En primer lugar, un espacio deteriorado, sucio, oscuro, desértico... influye en la sensación de inseguridad, 
en particular de las mujeres e indirectamente de los niños y niñas (Michaud, 2002; Morrell, 1998). Por otra parte, si partimos de que el espacio enseña, es decir, que a través del aspecto social de un lugar, el contexto, la calidad de luz y sonido, los objetos, etc. se interviene en el aprendizaje (Alderoqui, 2000), la falta de mantenimiento adecuado desvirtúa este proceso educativo. Si los pavimentos se deterioran, los ornamentos se estropean, si los árboles o parterres no se reponen, comienza un proceso de degradación y se pierde la eficacia educadora. En nuestra observación, los mismos niños reclaman en sus propuestas de mejora una mayor limpieza y un mejor estado de la vegetación.

\section{Espacios de socialización}

Los espacios públicos estudiados propician oportunidades para la interacción y la comunicación social entre personas que se conocen y entre personas extrañas entre sí. Así lo afirman las personas usuarias entrevistadas y las observaciones realizadas. Incluso en algunos casos, madres y padres de distintos barrios que se han conocido en los parques se llaman por teléfono para quedar en ellos y charlar. Como explicita Tonucci (2004) las nuevas formas de vida más autosuficientes e individualistas de los entornos urbanos hacen que a veces sintamos la necesidad de recrear nuevos vínculos sociales, nuevas formas de vecindario. Entonces los amigos son las personas que sacan el perro a la misma hora o aquellos que acompañan los niños a la misma escuela (p.67). Por su parte, Stevens (2004) considera que la concentración de una gran diversidad de personas desconocidas entre sí, objetos, significados y oportunidades para la acción en los espacios públicos urbanos, estimula el surgimiento de una gran variedad de actividades creativas, comportamientos y relaciones. Conversaciones, saludos con los vecinos, frases intercambiadas para preguntar o pedir alguna cosa ("¿está libre este banco?", "đtiene hora?"...), manifestaciones de afectividad (besos, abrazos...), contacto visual, miradas, etc., son algunas de las formas de interacción y de relación observadas entre los usuarios adultos e infantiles de los espacios públicos.

En las áreas de juegos infantiles los niños juegan y de esta forma aprenden a conocer su propio cuerpo y el espacio donde están jugando, a la vez que se relacionan con otras personas de su misma edad. En este sentido, es importante tener en cuenta que el entorno físico y el diseño de los espacios públicos son fundamentales para el desarrollo y la adquisición progresiva de autonomía. Una configuración clara y acotada del espacio público que limite el tráfico y favorezca el uso peatonal, con mezcla de usos y vigilancia natural del espacio fomenta el uso de las personas mayores y de los niños, avanzando la edad en que estos últimos pueden ir solos, reduciendo 
la necesidad de acompañantes que vigilen constantemente su juego (Sánchez de Madariaga, 2004). En nuestros casos de estudio, no es raro ver a niños (más que niñas) de más de 7 años sin acompañantes en las áreas de juego. No cabe duda que ganan en autonomía, independencia, seguridad, privacidad y autoestima.

Por otra parte, los niños tienen necesidades diversas, de movimiento y reposo, seguridad y desafio, socialización y autonomía, imitación y creación, imaginación y confrontación con la realidad, sentimiento y acción sobre las cosas (Alderoqui, 2000). Como hemos observado, los niños disfrutan al arrastrarse y trepar, subir y bajar y a veces disfrutan creando o imaginando cualquier cosa. Por lo tanto, quizás no sea tan necesario acercarles de todo, sino crearles foros donde la arquitectura sostenga la posibilidad de interacción y creación. Según Alderoqui (2000:2) "los 'buenos' espacios de aprendizaje son necesariamente 'desordenados', proveen un amplio rango de situaciones para realizar elecciones y opciones y dan oportunidades de crear su propio orden entre una variedad de elementos". Los espacios de juego deben invitar a la reacción. En nuestro caso, esta idea se puede manifestar en que los niños demandan, más que elementos concretos (como columpios o carriles bici) más espacio para jugar. También los expertos lo corroboran:

"Creo que faltan espacios vacíos. Espacio de campos donde no haya nada. Los niños/as cuando diseñan un espacio no ponen muchas cosas. (...) Hoy ya no tenemos lugares donde esconderse y a los niños/as les gusta mucho jugar y esconderse. Echo de menos laberintos de materiales sencillos." (Técnica de infancia, mujer,.... años).

Es por lo tanto, en la diversidad, calidad y en el mantenimiento de los espacios dónde se debe incidir para que cumplan con su función educadora y socializadora.

\section{Los conflictos como escuela de civismo}

La voluntad de incorporar este tema en nuestro análisis deriva más de la importancia que se le da en las fuentes teóricas consultadas, mayoritariamente procedentes del ámbito anglosajón, que de su centralidad en los discursos y las observaciones realizadas. Las actitudes y percepciones respecto al miedo y a la inseguridad no han aparecido jamás de manera espontánea, e incluso cuando se ha preguntado específicamente sobre este tema la reacción de nuestros interlocutores ha sido más de sorpresa o de negación que de constatación. Esto nos obliga a plantearnos futuras hipótesis: sería muy interesante conocer en qué medida esta diferencia en la centralidad del 
tema del miedo y la seguridad tiene relación con un determinado entorno urbano o con otras variables.

Los espacios estudiados son concebidos y percibidos en términos generales como espacios seguros y confortables. Aunque el miedo y la seguridad no sean temas centrales se han podido identificar algunos aspectos que podríamos situar en este marco de la seguridad y los conflictos. En realidad la seguridad de los niños en los espacios públicos y áreas de juego es una de las preocupaciones expresadas por los adultos entrevistados.

En primer lugar podemos citar el hecho de que la presencia de grupos de jóvenes en los espacios públicos en general o en los espacios de juego en particular, puede tener un efecto disuasorio sobre otros posibles usuarios potenciales, que rechazan el uso o buscan alternativas ante este hecho. Esta actitud preventiva no tiene porque tener relación con ningún incidente previo, sino que responde a los prejuicios de otros usuarios potenciales.

Un segundo aspecto que se ha detectado como problemático es la presencia de personas con perros. Aquí el conflicto surge cuando los perros invaden las áreas de juegos infantiles, tanto por la peligrosidad que pueden representar para los niños, como por la suciedad y los problemas de higiene y salud pública que generan con sus deposiciones.

El tercer foco de preocupación o el tercer conflicto de uso o de intereses es el que representan los coches y otros vehículos, es decir el tráfico rodado. Aquí la inseguridad se deriva de la presencia de vehículos circulando alrededor o muy cerca de las áreas de juego, especialmente cuando los niños son muy pequeños. Habría que añadir que la contaminación acústica y del aire generada por este elemento tiene también una incidencia negativa en la salud de los usuarios de los espacios de juego.

Finalmente se ha señalado al juego y concretamente a los propios elementos del espacio de juegos como generadores de peligros. A veces los problemas de seguridad también se asocian al diseño de los espacios, la presencia de barandillas, desniveles, escaleras... son vistos como problemáticos. La posibilidad de golpearse con un columpio, caer de la estructura o del tobogán, resbalar con el agua o simplemente caerse en el suelo son riesgos inherentes al hecho de jugar que las personas adultas a cargo de los niños no parecen estar dispuestos a enfrentar. Se produce una discordancia de intereses entre los adultos, que quieren juegos con las máximas garantías de seguridad, y los niños que buscan en el riesgo un estímulo del juego.

A la luz de estos conflictos también nos ha interesado observar qué tipo de estrategias o soluciones se han adoptado para enfrentarlos. En este sentido son revelado- 
ras las manifestaciones de los propios niños cuando apelan a la necesidad de preservar la convivencia, cuando reconocen que su derecho al juego tiene que ser compatible con el derecho al descanso de otras personas.

En relación a las diferentes situaciones planteadas hemos visto como los grupos de jóvenes a menudo ya optan por ocupar espacios más periféricos, menos visibles o incluso espacios alternativos, espacios "libres" que les eviten tener que competir por el espacio. Otra estrategia pasa por ocupar los espacios en tiempos alternativos a los de mayor uso, de ahí la tendencia de los jóvenes a colonizar el tiempo nocturno, aunque este hecho plantea nuevos conflictos relacionados con el ruido y el derecho al descanso del resto del vecindario, especialmente durante el verano cuando las ventanas están abiertas.

Para hacer frente a los problemas generados por el tráfico y por los perros se ha optado por cerrar los espacios de juegos infantiles instalando vallas bajitas que impidan que los perros entren y que al mismo tiempo impidan que los niños salgan del área controlada por los adultos. Cabe señalar que donde se ha adoptado esta solución se ha hecho por petición expresa de los usuarios y que este es un elemento ajeno al diseño inicial de los espacios en cuestión. Entre los aspectos negativos de estos cerramientos destacamos su efecto en la segregación de la población en el espacio, aunque los usuarios adultos lo valoran positivamente porque convierte su tarea de vigilancia en algo más relajado y placentero. Este objetivo también se consigue con la existencia de espacios intermedios que ejerzan un papel de barreras, como setos de vegetación, estanques... Sin embargo en ninguna ocasión las personas entrevistadas proponen una modificación de las normas de circulación en estos lugares (ej.: limitación de la velocidad) o la conversión del área de circulación en una zona peatonal, hecho que demuestra la demasiada permisividad que existe todavía en nuestras ciudades en cuanto al derecho de los vehículos frente al de los peatones. Esta voluntad de convertir las calles o los accesos al área de juegos en zonas peatonales, de prioridad invertida o de tráfico restringido es una opción que ya se ha llevado a cabo en alguno de los espacios estudiados.

En el tema del mobiliario de juego hay una regulación cada vez más estricta. Hay empresas especializadas que siguen la normativa europea y los ayuntamientos suelen acudir a ellas a la hora de equipar los espacios para asegurarse el mínimo de problemas al respecto. Este hecho convierte a los espacios de juego en áreas homogeneizadas en pro de la normativa europea y la seguridad de la infancia, pero a costa de la imaginación o el desarrollo de otros aspectos del aprendizaje o de la construcción de la identidad en relación a espacios singulares. 


\section{Políticas específicas y órganos de participación infantil}

En ninguno de los dos ayuntamientos consultados existe una política específica en materia de espacios de juegos. La situación actual es que diferentes áreas de la administración municipal, intervienen en este tema: urbanismo, medio ambiente, educación, mujeres, infancia, inmigración, deportes, cultura... pero la coordinación entre ellas no siempre es efectiva o simplemente no existe de manera formal. Ello no significa que puntualmente no haya buenas experiencias de colaboración, especialmente cuando hay buena sintonia y motivación personal tal como expresa una técnica de infancia: "Desde Vía Pública me llaman y me preguntan: ¿qué pensáis de esta calle que queremos arreglar? Por ejemplo yo he sido muy pesada pidiendo que se pongan charrancas en diferentes lugares de la ciudad".

Sin embargo, tanto los políticos como los técnicos consultados reconocen la necesidad y la eventual utilidad de esta política específica y/o esta coordinación. Por ejemplo se menciona la necesidad de establecer criterios técnicos, de tener en cuenta las diferentes edades, de proyectar espacios singulares, de fomentar el civismo, la cohesión social...

Manresa tiene entre sus proyectos de futuro a medio plazo elaborar un Plan de Infancia y Familia que contemplaría, entre otras, esta cuestión. En Sant Feliu de Llobregat el Plan de Mandato contempla la consolidación de los espacios de juego en los parques de la ciudad y se plantea como proyecto de futuro que la renovación del contrato de jardinería incluya el mantenimiento de los espacios de juego.

En cuanto a los órganos de participación de la población infantil Manresa no dispone de mecanismos participativos específicos para la infancia. Existen mecanismos de participación ciudadana de carácter general, como los Consejos Territoriales. Las asociaciones vecinales son otro de los canales de participación utilizados. Sin embargo en ambos casos no se contempla la participación directa de los niñas y las niñas.

Otro órgano de participación que podría intervenir es el Consejo de Mujeres, que también cuenta con representantes de las AMPAS (asociaciones de madres y padres). Sin embargo en este caso se ha visto que el tema de los espacios de juegos o incluso de los espacios públicos nunca ha sido tratado. La concejal responsable de los temas de igualdad constata la dificultad con que se encuentran para introducir el género en los temas urbanísticos: "Es como si todo lo que es más urbano no tocara, no queda dentro de los temas normales (...). Cuando entramos en las áreas más técnicas cuesta más llegar". 
En Sant Feliu de Llobregat sí existe desde hace unos años un Consell d'Infants (Consejo de Niños y Niñas). Es un grupo de trabajo de niños y niñas de entre $10 \mathrm{y}$ 11 años. La presencia de niños y niñas en el Consell no siempre es equilibrada, depende de los años, varía según cómo vayan las votaciones; sin embargo se tiene en cuenta que cuando haya que acudir a algún acto en representación del Consell d'Infants los representantes sean siempre un niño y una niña. El Consejo se reúne en el ayuntamiento una vez al mes para debatir sobre múltiples aspectos relacionados con su ciudad, proponer cambios para su mejora y preparar actividades y proyectos. Los espacios de juegos y los problemas relacionados con la movilidad peatonal en la ciudad son algunos de los temas tratados. El Consell d'Infants hace llegar sus proyectos a los representantes del consistorio, tanto al personal técnico como al político, y éstos evalúan sus propuestas. También reciben encargos por parte del consistorio para estudiar un determinado tema y elaborar informes o emitir recomendaciones. En la práctica se ha constatado que el Ayuntamiento es bastante sensible respecto de las propuestas del Consell d'Infants, lo cual ha permitido que éste se convierta en un interlocutor consolidado y consultado por servicios como urbanismo o movilidad.

\section{Conclusión}

Abordar el estudio de las geografías de la infancia nos ha permitido profundizar en uno de los campos más novedosos de la geografía social. Considerar la población infantil y/o juvenil como objeto de estudio desde nuestra disciplina supone ampliar las miras del campo de la investigación geográfica a través de la incorporación de nuevos grupos sociales y nuevos temas y problemas ligados a estas franjas de edad. Con ello conseguimos hacer visible un colectivo frecuentemente obviado o incluso conscientemente ausente en muchos estudios geográficos, que se supone que tienen en cuenta el conjunto de la población, cuando en realidad a menudo sólo han considerado a la población adulta, masculina, autóctona o de una determinada clase social.

De nuestro estudio sobre el papel de los espacios de juego en la vida cotidiana de los niños se desprenden algunas coincidencias con las investigaciones pioneras realizadas en otros contextos, en particular en los países anglosajones. El diseño de las áreas de juego, su contenido y la distribución de los elementos suele ser muy similar, independientemente del país, algo que puede responder a las directrices impuestas por la Unión Europea en materia de calidad y seguridad. Sin embargo, existen diferencias significativas relacionadas con el uso del espacio y con las preocupaciones y prioridades que sobre él manifiestan los usuarios y planificadores. 
Los espacios de juego tienen un papel esencial en la vida cotidiana de los niños de las ciudades estudiadas, hasta el punto que para muchos niños forman parte de sus geografías personales diarias (por lo general, ocupan los espacios y tiempos de transición entre la escuela y el hogar o las actividades en lugares cerrados). En este uso tan habitual de estos espacios puede encontrarse explicación en la tradición mediterránea de hacer parte de la vida en espacios públicos abiertos, un hecho que se acentúa en los núcleos de población medianos y pequeños en los que la gente se conoce y se trata de forma habitual 'en la calle'. Esta utilización que viene de lo 'tradicional' aumenta con la necesidad de vida al aire libre que imponen las actuales formas de vida, cada vez más desarrolladas en los ámbitos privados, en espacios cerrados y a menudo realizadas individualmente. En este sentido, muchos padres valoran de los espacios de juego en la ciudad la posibilidad de recreo al aire libre, de hacer 'salud' (a través del juego en movimiento) y el contacto con otros niños. Estas razones pueden explicar la intensidad de uso de los espacios de juego considerados, en particular los centrales, por concentrar mayor número de población, pero también los periféricos. Además, la imposición por parte de las normas urbanísticas actuales que asignan dentro del espacio público zonas de equipamiento con jardines y espacios lúdicos, indica la valoración positiva de estos lugares por la función ambiental, social y de calidad de vida que representan.

El factor edad es el elemento organizador de los espacios de juego considerados en el estudio. Los niños se apropian de los distintos lugares en función de la edad y el factor de género es significativo cuando se asocia a la edad. Es decir, a partir de los 7 u 8 años, la ocupación del espacio y el uso del mismo es distinto según sexos.

Los modelos de género son muy perceptibles en estos espacios de recreo: en el juego de los niños, en las personas que los acompañan y en lo que hacen, en el perfil de los otros usuarios... Y aunque son, en general, todavía espacios bastante feminizados, al ser lugares donde confluyen personas de distintas edades, sexos, clases y orígenes, constituyen excelentes laboratorios para observar y experimentar los roles y relaciones de género, y también para ponerlos en cuestión o para desafiarlos. Y éste es un elemento de interés en sociedades donde los roles de género se han transgredido muy poco y quizás menos en ciudades de pequeño tamaño.

La investigación en las dos ciudades y en distintas áreas dentro de la ciudad nos ha confirmado una vez más que el conocimiento es situado y que lo local es esencial para comprender cualquier manifestación de lo global. También se ha puesto de manifiesto que la geografía puede y debe ocuparse de la infancia en su relación con el espacio y el territorio, y que las categorias sociales de análisis como el género son fundamentales para comprender y actuar sobre esta relación. 


\section{Bibliografía}

Alderoqui, S. (2000): Los espacios públicos urbanos provocadores de aprendizaje para niños y jóvenes. En La ciudad que educa: dimensión pedagógica del urbanismo. Conferencia presentada en las III Jornadas Nacionales Enseñar a través de la ciudad y el museo, Mar de Plata, octubre, 2000 .

Michaud, A. (2002): La seguridad de las mujeres: de la dependencia a la autonomia. Montréal: Femmes et ville.

Morrell, H. (1998): Seguridad de las mujeres en la ciudad. En La vida de las mujeres en las ciudades. La ciudad, un espacio para el cambio. Madrid: Narcea ediciones, p. 131-145.

Sánchez de Madariaga, I. (2004): Urbanismo con perspectiva de género. Sevilla, Junta de Andalucía.

Stevens, Q. (2004): Urban escapades: play in Melbourne's public spaces. L. Lees (ed.) The emancipatory city? Paradoxes and possibilities. London: Sage Publications, pp. 139-157.

Borja, J. y Castells, M. (1997): Local y global. La gestión de las ciudades en la era de la información. Madrid, Taurus.

Droogleever, J. y Karsten, L. (1999) Contrastant polítiques: qüestions sobre emancipació, medi ambient i mobilitat. Documents d'Anàlisi Geogràfica, 35, p. 89-100.

Gagen, E.A. (2000): Playing the part. Performing gender in America's playgrounds. En S. Holloway y G. Valentine (eds.) Cbildren's geographies, Londres, Routledge, p. 213-229.

Karsten, L. (1998): Growing up in Amsterdam: differentiation and segregation in childre$n$ 's daily lives. Urban Studies, 35 (3), p. 565-581.
Karsten, L. (2002): Mapping childbood in Amsterdam: the spatial and social construction of children's domains in the city. Tijdschrift voor Economische en Sociale Geografie, 93 (3), p. 231-241.

Karsten, L. (2003): Children's use of public space: the gendered world of the playground. Childhood, 10, p. 457-473.

Matthews, H. (1995): Living on the edge: children as 'outsiders'. Tijdschrift voor Economische en Sociale Geografie, 86 (5), p. $456-466$.

McKendrick, JH; Bradford, MG. \& Fielder, AV. (2000): Time for a party! Making sense of the commercialisation of leisure space for children. En S. Holloway y G. Valentine (eds.) Cbildren's geograpbies, Londres, Routledge, p. 100-116.

Ortiz, A. (2003): Gènere, espais puiblics $i$ construcció del sentit de pertinenca a Barcelona (els barris de Prosperitat, el Verdum i el Raval). Tesi de doctorat. Departament de Geografia. Universitat Autònoma de Barcelona.

Paravicini, U. (2000): Rol y uso social de espacios públicos en una perspectiva de género. El renacimiento de la cultura urbana, Rosario, Municipalidad de Rosario.

Román, M. (1995): La reconstrucción del espacio cotidiano. Seminario "Tiempo y espacio en la vida de las mujeres". Santander: Universidad Menéndez Pelayo [página web consultada el 27 de abril de 2001].

Román, M. (2000): Niñ@s, ciudadan@s peligros@s. En Ciudades para un futuro más sostenible, Boletín $\mathrm{CF}+\mathrm{S}, 19$ [página web consultada el 22 de septiembre de 2002] http://habitat.aq.upm.es/boletin/n19/amr om.html]. 
Simpson, B. (1997): Towards the participation of children and young people in urban planning and design. Urban Studies, 34 (5-6), p. 907-925.

Tandy, C. (1999): Children's dimishing play space: a study of intergenerational change in children's use of their neighbourhoods. Australian Geographical Studies, 37 (2), p. 154-164.

Tonucci, F. (2004): Quan els infants diuen proa. Barcelona, Graó.

Valentine, G. (1996): Children should be seen and not heard: the production and trans- gression of adult's public space. Urban Geography, 17 (3), p. 205-220.

Valentine, G. (1997): 'Oh yes I can'. 'Oh no you can't': children and parents' understanding of kids' competence to negotiate public space safely. Antipode, 29 (1), p. 65-89.

Valentine, Gill (2002). People like us: negotiating sameness and difference in the research process. P. Moss (ed.) Feminist Geography in Practice: Research and metbods. Oxford: Blackwell, p. 116-126.

Valentine, G. (2004): Public space and the culture of childbood. Hants: Ashgate. 